ALGEBRAIC METHODS IN LOGIC AND IN COMPUTER SCIENCE

BANACH CENTER PUBLICATIONS, VOLUME 28

INSTITUTE OF MATHEMATICS

POLISH ACADEMY OF SCIENCES

WARSZAWA 1993

\title{
POLYADIC ALGEBRAS OVER NONCLASSICAL LOGICS
}

\author{
DON PIGOZZI \\ Department of Mathematics, Iowa State University \\ Ames, Iowa 50011, U.S.A. \\ E-mail:DPIGOZZI@IASTATE.EDU \\ ANTONINO SALIBRA \\ Dip. Informatica, University of Pisa \\ Corso Italia 40, I-56125 Pisa, Italy \\ E-mail: SALIBRA@DI.UNIPI.IT
}

\begin{abstract}
The polyadic algebras that arise from the algebraization of the first-order extensions of a SIC are characterized and a representation theorem is proved. Standard implicational calculi (SIC)'s were considered by H. Rasiowa [19] and include classical and intuitionistic logic and their various weakenings and fragments, the many-valued logics of Post and Łukasiewicz, modal logics that admit the rule of necessitation, BCK logic, etc.
\end{abstract}

Introduction. In [19] H. Rasiowa identifies a large class of propositional logics, the standard implicational calculi (SIC's), that can be algebraized in a meaningful way and which include most of the nonclassical logics considered in the literature: classical and intuitionistic logic and their various weakenings and fragments, the multiple-valued logics of Post and Łukasiewicz, the modal logics that admit the rule of necessitation, BCK logic, etc. The main purpose of this

1991 Mathematics Subject Classification: Primary 03G15; Secondary 03B22, 03B40, 08A05

Key words and phrases: intuitionistic logic, modal logic, many-valued logic, BCK logic, lambda calculus.

The work of the first author was supported in part by National Science Foundation Grant \#DMS 8805870.

The work of the second author was supported by a NATO Senior Fellowship Grant of the Italian Research Council.

The paper is in final form and no version of it will be published elsewhere. 
paper is to characterize the polyadic algebras that arise when the first-order extension of a SIC $\mathcal{S}$ is algebraized. Each $\mathrm{SIC} \mathcal{S}$ gives rise to the quasivariety of polyadic $\mathcal{S}$-algebras, which coincides with the polyadic Boolean algebras of Halmos [8] when $\mathcal{S}$ is classical propositional logic. The main result of the paper is a functional representation theorem for polyadic $\mathcal{S}$-algebras that comprehends many of the known representation results for polyadic algebras over nonclassical logics.

Polyadic and cylindric algebras and their representations have been considered by a number of authors and for various nonclassical logics, including intuitionistic logic: Georgescu [7], Kotas and Pieczkowski [2], Monk [14]; modal and tense logic: Freedman [4], Georgescu [5, 6]; multivalued logic: Schwartz [21]. We also obtain a representation theorem in each of these cases. However, our general representation theorem corresponds to what is called the functional representation theorem in Halmos [8, (10.9)], while the representation results in many of the above papers correspond more closely to Halmos's stronger representation for simple, locally finite polyadic Boolean algebras of infinite degree $[8,(17.3)]$. This point will be discussed more fully after we present our representation theorem in Section 3. For related but independent work see Diskin [3]. For an overview of the algebraization of quantifier logics see Németi [16].

Another purpose of the paper is to illustrate how the algebraization of nonclassical first-order predicate logic fits into a general framework of substitution and variable-binding that includes such diverse variable-binding operations as lambda abstraction, definite Riemann integration, universal and existential quantification (in both classical and intuitionistic logic), and various notions of generalized quantification that have been studied in model theory. An investigation of substitution and variable-binding in this general context has been initiated in [17]. The main conceptual device used is the abstract variable-binding calculus (VBC), which is modeled on the lambda calculus. Its characteristic feature is a set of variable-binding operations that generalize both lambda abstraction and quantification (but that, in contrast, can be specified to take multiple arguments and to simultaneously bind multiple variables). In the VBC, as in the lambda calculus and the usual formalizations of quantification logic, substitution of terms for variables is effected by physical replacement. In [17] an algebraic reformulation of the VBC is presented in which substitution is represented by an abstract operator. This gives rise to the class of polyadic $\Lambda$-structures, a universal Horn class. The polyadic $\mathcal{S}$-algebras for any SIC $\mathcal{S}$ form a special subclass. The main result of [17] is a functional representation theorem for polyadic $\Lambda$-structures, of which the representation theorem for polyadic $\mathcal{S}$-algebras given in the present paper is a special case.

Outline of paper. In the first section we summarize without proofs all definitions and results from [17] that will be needed in the subsequent part of the paper; in particular, the functional representation theorem for polyadic 
$\Lambda$-algebras is given in Thm. 1.12. Section 2 constitutes a self-contained exposition of the relevant part of the theory of SIC's and their first-order extensions; this material is taken directly from Rasiowa [19] with some changes in notation and terminology to better serve our purpose. All the new results of the paper appear in Section 3 where we define polyadic $\mathcal{S}$-algebras, discuss their connection with first-order logic over $\mathcal{S}$, and prove the main representation theorem (Thm. 3.7). The paper ends with a brief discussion of connections with other work and a proposal for future research.

The survey of metalogical results found in Section 2 is considerably more detailed than actually needed for the purposes of the paper, but we feel it is justified by the motivation it provides for the definitions and results of Section 3. It also provides us with the opportunity to present in concise form the basic elements of the theory of quantification over nonclassical logics. We hope the reader will find this useful.

1. Polyadic $(\Lambda, I)$-algebras. Let $I$ be a nonempty set. Following Halmos $[8]$ we call an arbitrary mapping $\sigma: I \rightarrow I$ a transformation (of $I$ ). The set of transformations is denoted by $I^{I}$. We use $\sigma, \tau, \lambda$ and $\pi$ to represent transformations, and $i$ and $j$ for elements of $I$. The identity transformation is denoted by $\iota$. For any subset $J$ of $I$ we write $\sigma J \tau$ to mean that $\sigma i=\tau i$ for all $i \in J$, and $\sigma J_{*} \tau$ to mean that $\sigma i=\tau i$ for all $i \notin J$; thus $\sigma J_{*} \tau$ iff $\sigma(I \backslash J) \tau$. J supports $\sigma$ if $\sigma J_{*} \iota$. $J$ is independent of $\sigma$ if $I \backslash J$ supports $\sigma . \sigma$ is of finite support if it has a finite support set. The set of transformations of finite support is denoted by $I^{(I)}$. We write $J \subseteq_{\omega} I$ to mean that $J$ is a finite subset of $I$, and $\operatorname{Sb}_{\omega} I$ is the set of all finite subsets of $I$.

Let $\Lambda=\langle\mathcal{N}, \mathcal{B}, \varrho\rangle$ be a first-order language with two disjoint sets $\mathcal{N}$ and $\mathcal{B}$ of operation symbols and an arity function $\varrho: \mathcal{N} \rightarrow \omega . \Lambda$ is called nonbinding or propositional if $\mathcal{B}=\emptyset$. Let $I$ be a nonempty set.

We consider a new first-order language that is closely related to $\Lambda$. Its nonlogical symbols fall into the following categories:

- An operation symbol $O$ of arity $\varrho O$ for each $O \in \mathcal{N}$. These are called nonbinding operations or propositional connectives.

- An $\mathrm{Sb}_{\omega} I$-indexed system of unary operation symbols $Q:=\left\langle Q_{J}: J \subseteq \subseteq_{\omega} I\right\rangle$ for each $Q \in \mathcal{B}$. These are called binding operations or generalized quantifiers.

- A unary operation symbol $S_{\sigma}$ for each $\sigma \in I^{(I)}$. These are called substitution operators.

Definition 1.1. Let $I$ be a nonempty set and $\Lambda$ a language in the above sense. By a polyadic $(\Lambda, I)$-algebra we mean an algebra of the form

$$
\boldsymbol{A}:=\left\langle A,\left\langle O^{\boldsymbol{A}}: O \in \mathcal{N}\right\rangle,\left\langle Q_{J}^{\boldsymbol{A}}: Q \in \mathcal{B}, J \subseteq_{\omega} I\right\rangle,\left\langle S_{\sigma}^{\boldsymbol{A}}: \sigma \in I^{(I)}\right\rangle\right\rangle
$$


that satisfies the following axioms (they are all assumed to be universally quantified):

$\left(\mathrm{PA}_{1}\right) \quad S_{\iota} x=x$

$\left(\mathrm{PA}_{2}\right) \quad S_{\sigma}\left(S_{\tau} x\right)=S_{\sigma \tau} x$, for all $\sigma, \tau \in I^{(I)}$;

$\left(\mathrm{PA}_{3}\right) \quad S_{\sigma} O\left(x_{1} \ldots x_{\varrho} O\right)=O\left(S_{\sigma} x_{1}, \ldots, S_{\sigma} x_{\varrho O}\right)$, for all $O \in \mathcal{N}, \sigma \in I^{(I)}$;

$\left(\mathrm{PA}_{4}\right) \quad S_{\sigma} Q_{J} x=S_{\tau} Q_{J} x$, for all $Q \in \mathcal{B}, J \subseteq \omega I$, and $\sigma, \tau \in I^{(I)}$ such that $\sigma J_{*} \tau$;

$\left(\mathrm{PA}_{5}\right) \quad Q_{J} S_{\sigma} x=S_{\sigma} Q_{\sigma^{-1}(J)} x$, for all $Q \in \mathcal{B}, J \subseteq_{\omega} I$, and $\sigma \in I^{(I)}$ such that $\sigma$ is one-to-one on $\sigma^{-1}(J)$.

$\boldsymbol{A}$ is a pseudomonotonic polyadic $(\Lambda, I)$-algebra if it satisfies the following pseudomonotonicity axiom.

$\left(\mathrm{PA}_{6}\right) \quad Q_{J} x=Q_{J} S_{\sigma} y \wedge Q_{J} y=Q_{J} S_{\tau} x \rightarrow Q_{J} x=Q_{J} y$, for all $Q \in \mathcal{B}, J \subseteq_{\omega} I$, and $\sigma, \tau \in I^{(I)}$ such that $\sigma J_{*} \iota$ and $\tau J_{*} \iota$.

$I$ is called the coordinate set and $\Lambda$ the base language of $\boldsymbol{A} . \boldsymbol{A}$ is infinitedimensional if $I$ is infinite. We refer to $\boldsymbol{A}$ as a polyadic $\Lambda$-algebra if $I$ is clear from context.

A polyadic $\Lambda$-algebra is a special case of the more general notion of a $\Lambda$ structure defined in [17]. In the latter a binding operation may be of arbitrary finite rank and the number of variables it simultaneously binds may be restricted. A $\Lambda$-structure may also have relations as well as nonbinding and binding operations.

Polyadic $\Lambda$-algebras and pseudomonotonic $\Lambda$-algebras are intended to generalize the polyadic Boolean algebras of Halmos [8] $\left({ }^{1}\right)$, with the nonbinding reduct of a polyadic $\Lambda$-algebra corresponding to the underlying Boolean algebra of a polyadic Boolean algebra. In particular, the nonbinding operations correspond to the Boolean operations $+, \cdot,-, 0$ and 1 . Axiom $\left(\mathrm{PA}_{3}\right)$ corresponds to the requirement, in the definition of polyadic Boolean algebras, that the substitution operations be endomorphisms of the underlying Boolean algebra. Completely missing in the definition of a polyadic $\Lambda$-algebra $\boldsymbol{A}$ are axioms specifying the structure of the $\mathcal{N}$-reduct of $\boldsymbol{A}$. In a polyadic Boolean algebra it is a Boolean algebra, but in a $\Lambda$-polyadic algebra it can be any algebra of logic, a Heyting or Lukasiewicz algebra for instance.

The single quantifier $\exists$ of polyadic Boolean algebras has been replaced by the family of binding operations. The axioms characterizing $\exists$ as a special kind of closure operation, in particular that it is monotonic with respect to the partial

$\left({ }^{1}\right)$ Strictly speaking, because of the restriction to finite subsets of $I$ and transformations of finite support in the axioms $\left(\mathrm{PA}_{1}\right)-\left(\mathrm{PA}_{5}\right)$, polyadic $\Lambda$-algebras are actually more closely related to quasipolyadic Boolean algebras. 
ordering of the underlying Boolean algebra, have been replaced by the pseudomonotonicity axiom $\left(\mathrm{PA}_{6}\right)$. Pseudomonotonicity appears to be the closest approximation to monotonicity that can be obtained without assuming a partial ordering on the underlying algebra. It corresponds roughly to Theorem 10.1 in [8] which asserts that, if $\boldsymbol{A}$ is a locally finite polyadic Boolean algebra of infinite degree, then

$$
S_{\sigma} \exists_{J} a=\bigvee\left\{S_{\lambda} a: \lambda J_{*} \sigma\right\}
$$

whenever $a \in A, J \subseteq I$, and $\sigma \in I^{I}$; the supremum indicated in the above formula is taken with respect to the lattice ordering of the underlying Boolean algebra of $\boldsymbol{A}$ and is extended over all transformations of $I$ such that $\lambda J_{*} \sigma\left(^{2}\right)$. To see that any locally finite polyadic Boolean algebra $\boldsymbol{A}$ is pseudomonotonic, suppose $\exists_{J} a=\exists_{J} S_{\sigma} b$ and $\exists_{J} b=\exists_{J} S_{\tau} a$ with $\sigma, \tau J_{*} \iota$. (Since $\boldsymbol{A}$ is locally finite we assume without loss of generality that $J$ is finite.) We have $\exists_{J} b=\bigvee\left\{S_{\lambda} b: \lambda J_{*} \iota\right\} \geq$ $S_{\sigma} b$. Using the fact that the existential quantifier is a closure operator on the underlying Boolean algebra of $\boldsymbol{A}$ we get

$$
\exists_{J} b=\exists_{J} \exists_{J} b \geq \exists_{J} S_{\sigma} b=\exists_{J} a .
$$

By symmetry, $\exists_{J} b \leq \exists_{J} a$, and hence $\exists_{J} a=\exists_{J} b$. So $\boldsymbol{A}$ is pseudomonotonic.

The pseudomonotonicity axiom is a quasi-identity. Thus the class of pseudomonotonic polyadic $\Lambda$-algebras form a quasivariety. The polyadic $\Lambda$-algebras, however, form a variety as do the polyadic Boolean algebras.

Support and independence. In the sequel $\boldsymbol{A}$ will represent a fixed but arbitrary polyadic $(\Lambda, I)$-algebra:

$$
\boldsymbol{A}:=\left\langle A, O^{\boldsymbol{A}}, Q_{J}^{\boldsymbol{A}}, S_{\sigma}^{\boldsymbol{A}}\right\rangle_{O \in \mathcal{N}, Q \in \mathcal{B}, J \subseteq_{\omega} I, \sigma \in I^{(I)}} .
$$

To simplify notation we normally omit the superscripts ${ }^{\boldsymbol{A}}$ when writing $O^{\boldsymbol{A}}, Q_{J}^{\boldsymbol{A}}$, and $S_{\sigma}^{\boldsymbol{A}}$.

$J \subseteq I$ supports an element $a$ of $\boldsymbol{A}$ if $S_{\sigma} a=S_{\tau} a$ for all $\sigma, \tau \in I^{(I)}$ such that $\sigma J \tau ; a$ is of finite support if it has a finite support set. $J$ is independent of $a$ if $I \backslash J$ supports $a . \boldsymbol{A}$ is locally finite if every element of $\boldsymbol{A}$ is of finite support.

The following results are all established in [17].

Proposition 1.2. Assume $\boldsymbol{A}$ is infinite-dimensional and $J \subseteq_{\omega} I$. If $S$ is finite and supports a, then $S \backslash J$ supports $Q_{J} a$.

Corollary 1.3. Assume $\boldsymbol{A}$ is infinite-dimensional and $J \subseteq_{\omega}$ I. If $S$ is finite and supports $a$, then $Q_{J} a=Q_{J \cap S} a$.

Proposition 1.4. Let $O \in \mathcal{N}$. If $S$ supports each of the elements $a_{1} \ldots a_{\varrho} O$, then it supports $O\left(a_{1} \ldots a_{\varrho} O\right)$.

$\left({ }^{2}\right)$ This theorem generalizes the well-known result of Rasiowa and Sikorski [20] to the effect that an existentially quantified formula $\exists x \varphi(x)$ of first-order logic is the supremum, in the natural lattice ordering of the Tarski-Lindenbaum algebra, of the set of all substitution instances of $\varphi(x)$. 
Proposition 1.5. Let $\sigma \in I^{(I)}$. If $S$ supports a, then $\sigma(S):=\{\sigma i: i \in S\}$ supports $S_{\sigma} a$.

Functional polyadic algebras. Let $S$ be an arbitrary set. We denote the set of all subsets of $S$ by $\operatorname{Sb}(S)$. Let $F: \operatorname{Sb}(S) \stackrel{\mathrm{p}}{\rightarrow} S$ be a partial second-order unary operation on $S$. We define a binary relation $\leq_{F}$ on the range $F(\operatorname{Sb}(S))$ as follows: $a \leq_{F} b$ iff there exist $X, Y \subseteq S$ such that $a=F(X)$ and $b=F(Y)$ and $X \subseteq Y$. The relation $\leq_{F}$ is obviously reflexive but, in general, neither transitive nor antisymmetric.

Definition 1.6. A partial second-order operation $F: \operatorname{Sb}(S) \stackrel{\mathrm{p}}{\rightarrow} S$ is said to be pseudomonotonic if $\leq_{F}$ is antisymmetric, i.e., $a=F(X)=F\left(X^{\prime}\right), b=F(Y)=$ $F\left(Y^{\prime}\right), X \subseteq Y \subseteq S$, and $Y^{\prime} \subseteq X^{\prime} \subseteq S$ imply $a=b$.

Let $\langle S, \leq\rangle$ be a partially ordered set. A second-order function $F: \operatorname{Sb}(S) \stackrel{\mathrm{p}}{\rightarrow} S$ is monotonic (antimonotonic) if $X \subseteq Y \subseteq S$ imply $F(X) \leq F(Y)$ (resp. $F(Y) \leq$ $F(X))$.

Observe that if $\langle S, \leq\rangle$ is a lattice and $F: \operatorname{Sb}(S) \stackrel{\mathrm{p}}{\rightarrow} S$ is either the generalized join or meet operation, then $F$ is respectively monotonic or antimonotonic.

The following result is obvious.

Proposition 1.7. Every monotonic or antimonotonic second-order partial operation on a partially ordered set is pseudomonotonic. In particular, the generalized meet and join operations on a lattice-ordered set are pseudomonotonic.

Definition 1.8. By a $\Lambda$-algebra of truth values, or simply a value $\Lambda$-algebra, we will mean an algebra

$$
\boldsymbol{V}=\left\langle V, O^{\boldsymbol{V}}, Q^{\boldsymbol{V}}\right\rangle_{O \in \mathcal{N}, Q \in \mathcal{B}}
$$

where $O^{\boldsymbol{V}}: V^{\varrho O} \rightarrow V$ is a $\varrho O$-ary operation on $V$ for each $O \in \mathcal{N}$, and $Q^{\boldsymbol{V}}$ : $\mathrm{Sb}(V) \stackrel{\mathrm{p}}{\rightarrow} V$ is a partial unary second-order operation on $V$ for each $Q \in \mathcal{B}$. $V$ is pseudomonotonic if $Q^{\boldsymbol{V}}$ is pseudomonotonic for each $Q \in \mathcal{B}$.

Definition 1.9. Let $\boldsymbol{V}$ be a value $\Lambda$-algebra and $D$ and $I$ nonempty sets. Define

$$
\overline{\boldsymbol{V}}:=\left\langle V^{D^{I}}, O^{\overline{\boldsymbol{V}}}, Q_{J}^{\overline{\boldsymbol{V}}}, S_{\sigma}^{\overline{\boldsymbol{V}}}\right\rangle_{O \in \mathcal{N}, Q \in \mathcal{B}, J \subseteq_{\omega} I, \sigma \in I^{(I)}}
$$

as follows:

(i) $\left(O^{\overline{\boldsymbol{V}}}\left(f_{1}, \ldots, f_{\varrho} O\right)\right)(p)=O^{\boldsymbol{V}}\left(f_{1}(p), \ldots, f_{\boldsymbol{\varrho} O}(p)\right)$ for all $f_{1}, \ldots, f_{\varrho} O \in V^{D^{I}}$ and $p \in D^{I}$; $p \in D^{I}$;

(ii) $\left(Q_{J}^{\bar{V}} f\right)(p)=Q^{\boldsymbol{V}}\left(\left\{f(q): q \in D^{I}, q J_{*} p\right\}\right)$, for all $f \in V^{D^{I}}, J \subseteq_{\omega} I$ and

(iii) $\left(S_{\sigma}^{\overline{\boldsymbol{V}}} b\right)(p)=f(p \circ \sigma)$, for all $f \in V^{D^{I}}$, for all $\sigma \in I^{(I)}$ and $p \in D^{I}$.

In the sequel $\overline{\boldsymbol{V}}$ will be denoted by $\boldsymbol{V}^{D^{I}}$; it is called the full partial functional polyadic $(\Lambda, I)$-algebra (over $D$ and $\boldsymbol{V}$ ). 
Observe that $Q_{J}^{\boldsymbol{A}} b$ is a partial function from $D^{I}$ to $\boldsymbol{V}$. D and $\boldsymbol{V}$ are respectively called the domain and the value algebra of $\boldsymbol{V}^{D^{I}}$. The universe $V$ of $\boldsymbol{V}$ is the set of truth values of $\boldsymbol{V}^{D^{I}}$.

Definition 1.10. A subalgebra $\boldsymbol{F}=\left\langle F, O^{\boldsymbol{F}}, Q_{J}^{\boldsymbol{F}}, S_{\sigma}^{\boldsymbol{F}}\right\rangle$ of $\boldsymbol{V}^{D^{I}}$ such that $Q_{J}^{\boldsymbol{F}} f$ is a total function from $D^{I}$ to $\boldsymbol{V}$ for all $f \in F$ is called a (total) functional polyadic $(\Lambda, I)$-algebra (over $D$ and $\boldsymbol{V})$.

The following two theorems are proved in [17].

THEOREM 1.11. Every functional polyadic $(\Lambda, I)$-algebra $\boldsymbol{F}$ is a $(\Lambda, I)$-polyadic algebra. If the value algebra of $\boldsymbol{F}$ is pseudomonotonic, then $\boldsymbol{F}$ is pseudomonotonic as a polyadic $(\Lambda, I)$-algebra.

In [17] we show how to interpret any theory of the untyped lambda calculus as a polyadic $\Lambda$-algebra (over an appropriate language $\Lambda$ ). Functional polyadic $\Lambda$-algebras correspond roughly to what are called environment models of the lambda calculus in Meyer [13] and syntactical interpretations in Barendregt [1]. A more comprehensive treatment of the untyped lambda calculus along the lines of polyadic algebras can be found in [18].

THEOREM 1.12 (Functional Representation Theorem). Every locally finite polyadic $(\Lambda, I)$-algebra $\boldsymbol{A}$ of infinite dimension is isomorphic to a functional polyadic $(\Lambda, I)$-algebra $\boldsymbol{F}$ whose set of truth values coincides with the universe $A$ of $\boldsymbol{A}$ and whose domain is equal to the coordinate set I. If $\boldsymbol{A}$ is pseudomonotonic, then the value algebra of $\boldsymbol{F}$ is pseudomonotonic.

More precisely, a locally finite polyadic $(\Lambda, I)$-algebra $\boldsymbol{A}$ is isomorphic to a subalgebra of $\boldsymbol{V}^{I^{I}}$ where

$$
\boldsymbol{V}=\left\langle V, O^{\boldsymbol{V}}, Q^{\boldsymbol{V}}\right\rangle_{O \in \mathcal{N}, Q \in \mathcal{B}}
$$

with $V=A, O^{\boldsymbol{V}}=O^{\boldsymbol{A}}$ for each $O \in \mathcal{N}$, and $Q^{\boldsymbol{V}}: \operatorname{Sb}(A) \stackrel{\mathrm{p}}{\rightarrow} A$ is defined for each $Q \in \mathcal{B}$ as follows: its domain is the set of all subsets of $A$ of the form $\left\{S_{\lambda} a: \lambda J_{*} \sigma\right\}$ for some $a \in A, J \subseteq \subseteq_{\omega} I$, and $\sigma \in I^{(I)}$. For such a set,

$$
Q^{\boldsymbol{V}}\left(\left\{S_{\lambda} a: \lambda J_{*} \sigma\right\}\right)=S_{\sigma}^{\boldsymbol{A}} Q_{J}^{\boldsymbol{A}} a .
$$

2. Algebraization of SIC logics. In the next section we will apply Thm. 1.12 to a wide class of polyadic algebras that arise from nonclassical logics. The logics we consider will be the first-order extensions of the so-called standard systems of implicational extensional propositional calculi (SIC's) considered by Rasiowa [19]. These include most of the nonclassical logics that have been studied in the literature: classical and intuitionistic logic and their various weakenings and fragments, the multiple-valued logics of Post and Lukasiewicz, and the modal logics that admit the rule of necessitation, BCK logic, etc.

In this section we survey both the algebraization of SIC's and the formal development of the corresponding first-order predicate calculi and their semantics. 
Apart from notation, we follow Rasiowa [19] with only minor technical changes. For us a logic $\mathcal{S}$ will be identified with a deductive system $\mathcal{S}=\left\langle\mathrm{Fos}_{\mathcal{S}}, \vdash_{\mathcal{S}}\right\rangle$, an ordered pair, where $\mathrm{Fo}_{\mathcal{S}}$ is the set of formulas of some formal language and $\vdash_{\mathcal{S}}$ is a binary relation that relates subsets of formulas to individual formulas such that the following conditions hold:

$$
\begin{gathered}
\Gamma \vdash_{\mathcal{S}} \alpha \text { for all } \alpha \in \Gamma, \\
\Gamma \vdash_{\mathcal{S}} \alpha \text { and } \Gamma \subseteq \Delta \text { imply } \Delta \vdash_{\mathcal{S}} \alpha, \\
\Gamma \vdash_{\mathcal{S}} \alpha \text { and } \Delta \vdash_{\mathcal{S}} \gamma \text { for every } \gamma \in \Gamma \text { imply } \Delta \vdash_{\mathcal{S}} \alpha, \\
\Gamma \vdash_{\mathcal{S}} \alpha \text { implies } \Delta \vdash_{\mathcal{S}} \alpha \text { for some } \Delta \subseteq_{\omega} \Gamma .
\end{gathered}
$$

Deductive systems are usually defined by axioms and rules of inference. An axiom can be any formula. An inference rule is a pair $\langle\Gamma, \alpha\rangle$ where $\Gamma \subseteq_{\omega}$ Fos and $\alpha \in \mathrm{Fo}_{\mathcal{S}}$; we normally write such a rule in the form $\frac{\Gamma}{\alpha}$. Let Ax and In be sets of axioms and inference rules. A formula $\alpha$ is derivable by Ax and In from a set $\Gamma$ of formulas, in symbols $\Gamma \vdash_{\text {Ax,In }} \alpha$, if $\alpha$ is contained in every set $\Delta$ of formulas that includes Ax and is closed under all inference rules $\frac{\Theta}{\beta}$ in the sense that $\beta \in \Delta$ whenever $\Theta \subseteq \Delta .\left\langle\mathrm{Fo}_{\mathcal{S}}, \vdash_{\mathrm{Ax}, \mathrm{In}}\right\rangle$ is a deductive system, and it is not difficult to see that every deductive system $\mathcal{S}$ is of this form; Ax and In collectively are called an axiomatization of $\mathcal{S}$.

A deductive system $\mathcal{S}=\left\langle\mathrm{Fo}_{\mathcal{S}}, \vdash_{\mathcal{S}}\right\rangle$ is propositional if $\mathrm{Fo}_{\mathcal{S}}$ is the set of formulas of a propositional (i.e., nonbinding) language $\Lambda=\langle\mathcal{N}, \varrho\rangle$. For a propositional system $\mathcal{S}$ over a language $\Lambda$ we write $\mathcal{S}=\left\langle\Lambda, \vdash_{\mathcal{S}}\right\rangle$ and denote the set of formulas by $\mathrm{Fo}_{\Lambda}$ instead of $\mathrm{Fo}_{\mathcal{S}} . \mathrm{Fo}_{\Lambda}$ contains a denumerable set $p_{0}, p_{1}, p_{2}, \ldots$ of propositional variables and, for each propositional connective $O \in \mathcal{N}$ and all $\alpha_{1}, \ldots, \alpha_{\varrho} O \in \mathrm{Fo}_{\Lambda}$, $O \alpha_{1} \ldots \alpha_{\varrho} O \in \mathrm{Fo}_{\Lambda}$. A propositional system $\mathcal{S}$ is structural if $\Gamma \vdash_{\mathcal{S}} \alpha$ implies $\{h \gamma$ : $\gamma \in \Gamma\} \vdash_{\mathcal{S}} h \alpha$ for every substitution $h$ of arbitrary formulas for propositional variables.

A SIC is a structural propositional $\operatorname{logic} \mathcal{S}=\left\langle\Lambda, \vdash_{\mathcal{S}}\right\rangle$ with a special binary connective $\Rightarrow$ and a propositional constant $\top$ satisfying the following six conditions for all $\alpha, \beta, \gamma \in \mathrm{Fo}_{\Lambda}$ :

$$
\begin{gathered}
\vdash_{\mathcal{S}} \top, \\
\vdash_{\mathcal{S}} \alpha \Rightarrow \alpha, \\
\vdash_{\mathcal{S}} \alpha \Rightarrow \beta \text { and } \vdash_{\mathcal{S}} \alpha \text { imply } \vdash_{\mathcal{S}} \beta, \\
\vdash_{\mathcal{S}} \alpha \Rightarrow \beta \text { and } \vdash_{\mathcal{S}} \beta \Rightarrow \gamma \text { imply } \vdash_{\mathcal{S}} \alpha \Rightarrow \gamma, \\
\vdash_{\mathcal{S}} \alpha \text { implies } \vdash_{\mathcal{S}} \beta \Rightarrow \alpha, \\
\alpha_{1} \equiv_{\Delta} \beta_{1}, \ldots, \alpha_{\varrho} \equiv_{\Delta} \beta_{\varrho} O \text { imply } O \alpha_{1} \ldots \alpha_{\varrho} O \equiv_{\Delta} O \beta_{1} \ldots \beta_{\varrho} O \text { for all } O \in \mathcal{N} .
\end{gathered}
$$

In the last condition $\alpha \equiv \Delta \beta$ denotes the conjunction of the two entailments $\Delta \vdash_{\mathcal{S}} \alpha \Rightarrow \beta$ and $\Delta \vdash_{\mathcal{S}} \beta \Rightarrow \alpha$. To simplify notation we assume in the sequel that $\Rightarrow$ and $\top$ have been separated from $\mathcal{N}$. 
Every SIC is algebraizable in the sense of [2, Def. 2.10] (apply [2, Cor. 4.8]). Its equivalent algebraic semantics ([2, Def. 2.8]) turns out to be exactly the quasivariety of $\mathcal{S}$-algebras defined in Rasiowa [19]:

Definition 2.1. An algebra of truth values for $\mathcal{S}$, more simply an $\mathcal{S}$-algebra, is a $\Lambda$-algebra $\boldsymbol{V}=\left\langle V, \Rightarrow^{\boldsymbol{V}}, \top^{\boldsymbol{V}}, O^{\boldsymbol{V}}\right\rangle_{O \in \mathcal{N}}$ defined by the following identities and quasi-identities:

$\left(\mathrm{SA}_{1}\right) \quad \alpha=\top$ for each axiom $\alpha$ of $\mathcal{S}$;

$\left(\mathrm{SA}_{2}\right) \quad \alpha_{1}=\top \wedge \ldots \wedge \alpha_{n}=\top \rightarrow \beta=\top$, for each inference rule $\frac{\alpha_{1}, \ldots, \alpha_{n}}{\beta}$ of $\mathcal{S}$;

$\left(\mathrm{SA}_{3}\right) \quad(x \Rightarrow y)=\top \wedge(y \Rightarrow z)=\top \rightarrow(x \Rightarrow z)=\top$;

$\left(\mathrm{SA}_{4}\right) \quad(x \Rightarrow y)=\top \wedge(y \Rightarrow x)=\top \rightarrow x=y$.

The universe $V$ of $\boldsymbol{V}$ is called the set of truth values.

This definition can be found in [19, VIII.6]. That $\mathcal{S}$-algebras form the equivalent algebraic semantics for $\mathcal{S}$ follows easily from [2, Thm. 2.17, Cor. 4.8].

Rasiowa proves the following completeness theorem for every SIC $\mathcal{S}$ : Let $\alpha$ be any $\Lambda$-formula. Then $\vdash_{\mathcal{S}} \alpha$ iff $\alpha=\top$ is an identity of $\mathcal{S}$-algebras [19, VIII.7.1]. Implicit in [19] is the following stronger completeness theorem (it can also be obtained from [2, Def. 2.8] by using the fact that $\mathcal{S}$-algebras form an equivalent algebraic semantics for $\mathcal{S}$ ): Let $\alpha_{1}, \ldots, \alpha_{n}, \beta$ be a finite sequence of $\Lambda$-formulas; then

$$
\alpha_{1}, \ldots, \alpha_{n} \vdash_{\mathcal{S}} \beta \quad \text { iff } \quad \alpha_{1}=\top \wedge \ldots \wedge \alpha_{n}=\top \rightarrow \beta=\top
$$

is a quasi-identity of $\mathcal{S}$-algebras .

It follows from the definition that $(x \Rightarrow x)=\top$ is an identity of every $\mathcal{S}$-algebra [19, VIII.6.2]. Combined with the quasi-identities $\left(\mathrm{SA}_{3}\right)$ and $\left(\mathrm{SA}_{4}\right)$ this shows that the relation $\leq$ defined by

$$
u \leq v \quad \text { iff } \quad\left(u \Rightarrow^{\boldsymbol{V}} v\right)=\top^{\boldsymbol{V}}
$$

is a partial ordering relation on every $\mathcal{S}$-algebra $\boldsymbol{V}$. We call this the implication ordering on $\boldsymbol{V}$.

First-order predicate SIC's. Generalizing Henkin [10] and Mostowski [15], Rasiowa [19] defines the first-order extension $\mathcal{S}^{*}$ of an arbitrary SIC $\mathcal{S}$ as follows: The language $\Lambda^{*}=\langle\mathcal{N}, \mathcal{B}, \varrho\rangle$ is obtained from $\Lambda=\langle\mathcal{N}, \varrho\rangle$ by adjoining two binding operations $\forall$ and $\exists$; thus $\mathcal{B}=\{\forall, \exists\}$. A fixed denumerable set $\mathrm{Va}=\left\{v_{i}\right.$ : $i<\omega\}$ of individual variables is designated. Sets $\mathcal{P}$ of predicates and $\mathcal{F}$ of function symbols are also designated. The sets $\mathrm{Te}_{\Lambda^{*}}$ of $\Lambda^{*}$-terms and $\mathrm{Fo}_{\Lambda^{*}}$ of $\Lambda^{*}$-formulas are defined in the usual way.

The first-order deductive system $\mathcal{S}^{*}=\left\langle\Lambda^{*}, \vdash \mathcal{S}^{*}\right\rangle$ associated with $\mathcal{S}$ is defined by the following axioms and rules of inference:

- $\alpha\left(\varphi_{1}, \ldots, \varphi_{k}\right)$, for each axiom $\alpha\left(p_{1}, \ldots, p_{k}\right)$ of $\mathcal{S}$ and all $\varphi_{1}, \ldots, \varphi_{k} \in \mathrm{Fo}_{\Lambda^{*}}$. 
- $\frac{\alpha_{1}\left(\varphi_{1}, \ldots, \varphi_{k}\right), \ldots, \alpha_{n}\left(\varphi_{1}, \ldots, \varphi_{k}\right)}{\beta\left(\varphi_{1}, \ldots, \varphi_{k}\right)}$, for each inference rule $\frac{\alpha_{1}\left(p_{1}, \ldots, p_{k}\right), \ldots, \alpha_{n}\left(p_{1}, \ldots, p_{k}\right)}{\beta\left(p_{1}, \ldots, p_{k}\right)}$ of $\mathcal{S}$ and all $\varphi_{1}, \ldots, \varphi_{k} \in \mathrm{Fo}_{\Lambda^{*}}$.

- Rule of substitution for free variables: $\frac{\varphi\left(v_{i_{1}}, \ldots, v_{i_{k}}\right)}{\varphi\left(t_{1}, \ldots, t_{k}\right)}$, for $\varphi\left(v_{i_{1}}, \ldots, v_{i_{k}}\right) \in \mathrm{Fo}_{\Lambda^{*}}$ and all $t_{1}, \ldots, t_{k} \in \mathrm{Te}_{\Lambda^{*}}$ such that the $t_{1}, \ldots, t_{k}$ are free for $v_{i_{1}}, \ldots, v_{i_{k}}$ in $\varphi$.

- Rule of introduction of the universal quantifier: $\frac{\varphi \Rightarrow \psi\left(v_{i}\right)}{\varphi \Rightarrow \forall v_{j} \psi\left(v_{j}\right)}$, for all $\varphi, \psi \in \mathrm{Fo}_{\Lambda^{*}}$ such that $v_{i}$ does not occur free in $\varphi$ and $v_{j}$ is free for $v_{i}$ in $\psi$.

- Rule of elimination of the universal quantifier: $\frac{\varphi \Rightarrow \forall v_{j} \psi\left(v_{j}\right)}{\varphi \Rightarrow \psi\left(v_{i}\right)}$, for all $\varphi, \psi \in \mathrm{Fo}_{\Lambda^{*}}$ such that $v_{j}$ is free for $v_{i}$ in $\psi$.

- Rule of introduction of the existential quantifier: $\frac{\varphi\left(v_{i}\right) \Rightarrow \psi}{\exists v_{j} \varphi\left(v_{j}\right) \Rightarrow \psi}$, for all $\varphi, \psi \in \mathrm{Fo}_{\Lambda^{*}}$ such that $v_{i}$ does not occur free in $\psi$ and $v_{j}$ is free for $v_{i}$ in $\varphi$.

- Rule of elimination of the existential quantifier: $\frac{\exists v_{j} \varphi\left(v_{j}\right) \Rightarrow \psi}{\varphi\left(v_{i}\right) \Rightarrow \psi}$, for all $\varphi, \psi \in \mathrm{Fo}_{\Lambda^{*}}$ such that $v_{j}$ is free for $v_{i}$ in $\varphi$.

Semantics of first-order SIC's. The completeness theorem. Let $\mathcal{S}$ be a SIC over the propositional language $\Lambda$. A partial $\mathcal{S}$-realization of the first-order language $\Lambda^{*}$ associated with $\Lambda$ is a system $\boldsymbol{R}=\left\langle R, \boldsymbol{V}, F^{\boldsymbol{R}}, P^{\boldsymbol{R}}\right\rangle_{F \in \mathcal{F}, P \in \mathcal{P}}$ such that $R$ is a nonempty set, $\boldsymbol{V}$ is an arbitrary $\mathcal{S}$-algebra, $F^{\boldsymbol{R}}: R^{\boldsymbol{\varrho} F} \rightarrow R$, and $P^{\boldsymbol{R}}: R^{\boldsymbol{\rho} P} \rightarrow V$, where $V$ is the set of truth values of $\boldsymbol{V}$.

An assignment for $\boldsymbol{R}$ is a mapping $\boldsymbol{r}:\left\{v_{n}: n<\omega\right\} \rightarrow R$. It can be uniquely extended to a realization function for the set of all $\Lambda^{*}$-terms, which will also be denoted by $\boldsymbol{r}$. For any assignment $\boldsymbol{r}, j<\omega$, and $r \in R, \boldsymbol{r}\left(r / v_{j}\right)$ will denote the new assignment such that

$$
\boldsymbol{r}\left(r / v_{j}\right)\left(v_{i}\right)= \begin{cases}r & \text { if } i=j, \\ \boldsymbol{r}\left(v_{i}\right) & \text { otherwise. }\end{cases}
$$

The realization function $\llbracket-\rrbracket_{\boldsymbol{r}}^{\boldsymbol{R}}: \mathrm{Fo}_{\Lambda^{*}} \rightarrow V$ for the set of all $\Lambda^{*}$-formulas is now defined by recursion in essentially the same way satisfaction is defined for classical two-valued logic:

- $\llbracket P t_{1} \ldots t_{\boldsymbol{\varrho} P} \rrbracket_{\boldsymbol{r}}^{\boldsymbol{R}}=P^{\boldsymbol{R}}\left(\boldsymbol{r}\left(t_{1}\right), \ldots, \boldsymbol{r}\left(t_{\boldsymbol{\varrho}} P\right)\right)$ for every atomic formula $P t_{1} \ldots t_{\boldsymbol{\varrho} P}$.

- $\llbracket O \varphi_{1} \ldots \varphi_{\varrho} O \rrbracket_{\boldsymbol{r}}^{\boldsymbol{R}}=O^{\boldsymbol{V}}\left(\llbracket \varphi_{1} \rrbracket_{\boldsymbol{r}}^{\boldsymbol{R}}, \ldots, \llbracket \varphi_{\varrho} O \rrbracket_{\boldsymbol{r}}^{\boldsymbol{R}}\right)$ for every $O \in \mathcal{N}$ and all $\varphi_{1}, \ldots, \varphi_{\varrho} O$ $\in \mathrm{Fo}_{\Lambda^{*}}$, provided each $\llbracket \varphi_{i} \rrbracket_{\boldsymbol{r}}^{\boldsymbol{R}}$ is defined; otherwise it is undefined.

In the next two parts of the definition, the generalized meet $\bigwedge$ and generalized $\bigvee$ are both taken with respect to the implication ordering of $\boldsymbol{V}$.

- $\llbracket \forall v_{i} \varphi\left(v_{i}\right) \rrbracket_{\boldsymbol{r}}^{\boldsymbol{R}}=\bigwedge_{r \in R} \llbracket \varphi\left(v_{i}\right) \rrbracket_{\boldsymbol{r}\left(r / v_{i}\right)}^{\boldsymbol{R}}$ for all $\varphi\left(v_{i}\right) \in \mathrm{Fo}_{\Lambda^{*}}$ and $i<\omega$, provided $\llbracket \varphi\left(v_{i}\right) \rrbracket_{\boldsymbol{r}\left(r / v_{i}\right)}^{\boldsymbol{R}}$ exists for every $r \in R$ along with the generalized meet; otherwise it is undefined. 
- $\llbracket \exists v_{i} \varphi\left(v_{i}\right) \rrbracket_{\boldsymbol{r}}^{\boldsymbol{R}}=\bigvee_{r \in R} \llbracket \varphi\left(v_{i}\right) \rrbracket_{\boldsymbol{r}\left(r / v_{i}\right)}^{\boldsymbol{R}}$ for all $\varphi\left(v_{i}\right) \in \mathrm{Fo}_{\Lambda^{*}}$ and $i<\omega$, provided $\llbracket \varphi\left(v_{i}\right) \rrbracket_{\boldsymbol{r}\left(r / v_{i}\right)}^{\boldsymbol{R}}$ exists for every $r \in R$ along with the generalized join; otherwise it is undefined.

The following lemma is easily proved by induction on the structure of formulas.

LEMma 2.2. Let $\varphi\left(v_{i_{1}}, \ldots, v_{i_{k}}\right) \in \mathrm{Fo}_{\Lambda^{*}}$ and let $v_{j_{1}}, \ldots, v_{j_{k}}$ be variables that do not occur bound in $\varphi$. Then for every assignment $\boldsymbol{r}$

$$
\left.\llbracket \varphi\left(v_{j_{1}}, \ldots, v_{j_{k}}\right) \rrbracket_{\boldsymbol{r}}^{\boldsymbol{R}}=\llbracket \varphi\left(v_{i_{1}}, \ldots, v_{i_{k}}\right) \rrbracket_{\boldsymbol{r}\left(\boldsymbol{r}\left(v_{j_{1}}\right) / v_{i_{1}}\right) \ldots\left(\boldsymbol{r}\left(v_{j_{k}}\right) / v_{i_{k}}\right)}^{\boldsymbol{R}}\right) .
$$

A partial $\mathcal{S}$-realization is called a total $\mathcal{S}$-realization (or simply an $\mathcal{S}$-realization) if $\llbracket \varphi \rrbracket_{\boldsymbol{r}}^{\boldsymbol{R}}$ is defined for every $\varphi \in \mathrm{Fo}_{\Lambda^{*}}$ and every assignment $\boldsymbol{r}$. If $\llbracket \psi \rrbracket_{\boldsymbol{r}}^{\boldsymbol{R}}=\top^{\boldsymbol{R}}$ for every assignment $\boldsymbol{r}$, we write $\llbracket \psi \rrbracket^{\boldsymbol{R}}=T^{\boldsymbol{R}}$. Define the relation $\vDash_{\mathcal{S}}$ by

$$
\varphi_{1}, \ldots, \varphi_{n} \vDash_{\mathcal{S}} \psi \quad \text { iff } \quad \llbracket \varphi_{1} \rrbracket^{\boldsymbol{R}}=\ldots=\llbracket \varphi_{n} \rrbracket^{\boldsymbol{R}}=\top^{\boldsymbol{R}} \text { imply } \llbracket \psi \rrbracket^{\boldsymbol{R}}=\top^{\boldsymbol{R}}
$$

for every $\mathcal{S}$-realization $\boldsymbol{R}$.

The following completeness theorem can be found in [19, Supplement 11.1, 11.2, 12.4].

Theorem 2.3. Let $\mathcal{S}$ be a SIC over a language $\Lambda$, and let $\mathcal{F}$ and $\mathcal{P}$ be any sets of function and predicate symbols. For all $\varphi_{1}, \ldots, \varphi_{n}, \psi \in \mathrm{Fo}_{\Lambda^{*}}$,

$$
\varphi_{1}, \ldots, \varphi_{n} \vdash_{\mathcal{S}} \psi \quad \text { iff } \varphi_{1}, \ldots, \varphi_{n} \vDash_{\mathcal{S}} \psi .
$$

3. Polyadic $\mathcal{S}$-algebras and their representation. Let $\mathcal{S}$ be a fixed but arbitrary SIC over the language $\Lambda=\langle\mathcal{N}, \varrho\rangle$, and let $\mathrm{Va}=\left\{v_{i}: i<\omega\right\}$ be a fixed, denumerable set of individual variables. We call an $\mathcal{S}$-realization restricted if there are no function symbols, i.e., $\mathcal{F}=\emptyset$. Let $\boldsymbol{R}=\left\langle R, \boldsymbol{V}, P^{\boldsymbol{R}}\right\rangle_{P \in \mathcal{P}}$ be a restricted $\mathcal{S}$-realization, where $\boldsymbol{V}=\left\langle V, \Rightarrow^{\boldsymbol{V}}, \top^{\boldsymbol{V}}, O^{\boldsymbol{V}}\right\rangle_{O \in \mathcal{N}}$ is the algebra of truth values of $\boldsymbol{R}$, an $\mathcal{S}$-algebra. $\boldsymbol{R}$ gives rise in a natural way to a functional polyadic $\left(\Lambda^{*}, \omega\right)$-algebra $\boldsymbol{F}(\boldsymbol{R})$ in essentially the same way a classical first-order relational structure gives rise to a set polyadic Boolean algebra or a set cylindric algebra (see [11, Part I, p. 9]). The domain of $\boldsymbol{F}(\boldsymbol{R})$ is $R$ and its value algebra is $\boldsymbol{V}^{*}=\left\langle V, \Rightarrow^{\boldsymbol{V}}, \top^{\boldsymbol{V}}, O^{\boldsymbol{V}}, \forall \boldsymbol{V}^{*}, \exists^{\boldsymbol{V}^{*}}\right\rangle_{O \in \mathcal{N}}$, where the partial second-order functions $\forall \boldsymbol{V}^{*}, \exists^{\boldsymbol{V}^{*}}: \mathrm{Sb}(V) \stackrel{\mathrm{p}}{\rightarrow} V$ are defined as follows:

$$
\begin{aligned}
& \forall V^{*}(X)= \begin{cases}\wedge X & \text { if the generalized meet exists } \\
\text { undefined } & \text { otherwise }\end{cases} \\
& \exists \boldsymbol{V}^{*}(X)= \begin{cases}\bigvee X & \text { if the generalized join exists } \\
\text { undefined } & \text { otherwise }\end{cases}
\end{aligned}
$$

As before, the generalized meet and join are taken with respect to the implication ordering of $\boldsymbol{V}$.

Consider the full partial functional polyadic $\left(\Lambda^{*}, \omega\right)$-algebra $\left(\boldsymbol{V}^{*}\right)^{R^{\omega}}$ (Def. 1.9). Each assignment $\boldsymbol{r}$ for $\boldsymbol{R}$ can be associated in a natural way with an element $\boldsymbol{r}^{\prime}$ of $R^{\omega}$, viz., $\boldsymbol{r}^{\prime}(i)=\boldsymbol{r}\left(v_{i}\right)$ for each $i<\omega$; in the sequel we identify these two 
functions. In a similar manner every predicate $P^{\boldsymbol{R}}$ of $\boldsymbol{R}$ can be identified with the element $P^{\prime}$ of $\left(\boldsymbol{V}^{*}\right)^{R^{\omega}}$ where

$$
P^{\prime}(\boldsymbol{r})=P^{\boldsymbol{R}}(\boldsymbol{r}(0), \ldots, \boldsymbol{r}(\varrho P-1))\left(=\llbracket P v_{0} \ldots v_{\boldsymbol{\varrho} P-1} \rrbracket_{\boldsymbol{r}}^{\boldsymbol{R}}\right) .
$$

Definition 3.1. $\quad \boldsymbol{F}(\boldsymbol{R})$ is the subalgebra of $\left(\boldsymbol{V}^{*}\right)^{R^{\omega}}$ generated by the set $\left\{P^{\boldsymbol{R}}: P \in \mathcal{P}\right\}$.

LEMMA 3.2. $\boldsymbol{F}(\boldsymbol{R})$ is a functional polyadic $\left(\Lambda^{*}, \omega\right)$-algebra; i.e., $\forall_{J}^{\boldsymbol{F}(\boldsymbol{R})}$ and $\exists_{J}^{\boldsymbol{F}(\boldsymbol{R})}$ are defined for all $J \subseteq \omega \omega$.

Proof. We show first of all that for each $f \in F(\boldsymbol{R})$, there exists a $\varphi \in \mathrm{Fo}_{\Lambda^{*}}$ such that $f(\boldsymbol{r})=\llbracket \varphi \rrbracket_{\boldsymbol{r}}^{\boldsymbol{R}}$ for each $\boldsymbol{r} \in R^{\omega}$. This is proved by showing that the subset of $F(\boldsymbol{R})$ consisting of all elements of the form $\llbracket \varphi \rrbracket^{\boldsymbol{R}}$ contains $P^{\boldsymbol{R}}$ for each $P \in \mathcal{P}$ and is closed under the operations of $\boldsymbol{F}(\boldsymbol{R})$. For illustration we verify the closure under substitution and universal quantification.

Suppose $f=\llbracket \varphi \rrbracket^{\boldsymbol{R}}$ and $\sigma \in \omega^{(\omega)}$, say $\sigma=\left(j_{1} / i_{1}\right) \ldots\left(j_{k} / i_{k}\right)$. Write $\varphi$ in the form $\varphi\left(v_{i_{1}}, \ldots, v_{i_{k}}\right)$; we assume without loss of generality that $v_{j_{1}}, \ldots, v_{j_{k}}$ do not occur bound in $\varphi$. For all $\boldsymbol{r} \in R^{\omega}$,

$$
\begin{aligned}
\left(S_{\sigma}^{\boldsymbol{F}(\boldsymbol{R})} f\right)(\boldsymbol{r}) & =f(\boldsymbol{r} \circ \sigma) \quad \text { by Def. } 1.9 \\
& =\llbracket \varphi\left(v_{i_{1}}, \ldots, v_{i_{k}}\right) \rrbracket_{\boldsymbol{r} \circ \sigma}^{\boldsymbol{R}} \\
& =\llbracket \varphi\left(v_{i_{1}}, \ldots, v_{i_{k}}\right) \rrbracket_{\boldsymbol{r}\left(\boldsymbol{r}\left(j_{1}\right) / i_{1}\right) \ldots\left(\boldsymbol{r}\left(j_{k}\right) / i_{k}\right)}^{\boldsymbol{R}} .
\end{aligned}
$$

Thus, by Lemma 2.2,

$$
S_{\sigma}^{\boldsymbol{F}(\boldsymbol{R})} f=\llbracket \varphi\left(v_{j_{1}}, \ldots, v_{j_{k}}\right) \rrbracket_{\boldsymbol{r}}^{\boldsymbol{R}} .
$$

Suppose now that $f=\llbracket \varphi \rrbracket^{\boldsymbol{R}}$ and $J=\left\{i_{1}, \ldots, i_{k}\right\}$. We will show that

$$
\forall_{J}^{\boldsymbol{F}(\boldsymbol{R})} f=\llbracket \forall v_{i_{1}} \ldots \forall v_{i_{k}} \varphi \rrbracket^{\boldsymbol{R}} \text {. }
$$

Let $\boldsymbol{r} \in R^{\omega}$. Then

$$
\begin{aligned}
\left(\forall_{J}^{\boldsymbol{F}(\boldsymbol{R})} f\right)(\boldsymbol{r})= & \bigwedge\left\{f(\boldsymbol{s}): \boldsymbol{s} \in R^{\omega}, \boldsymbol{s} J_{*} \boldsymbol{r}\right\} \quad \text { by Def. } 1.9 \\
= & \bigwedge\left\{\llbracket \varphi \rrbracket_{\boldsymbol{s}}^{\boldsymbol{R}}: \boldsymbol{s} \in R^{\omega}, \boldsymbol{s} J_{*} \boldsymbol{r}\right\} \\
= & \bigwedge\left\{\llbracket \varphi \rrbracket_{\boldsymbol{r}\left(\boldsymbol{s}\left(i_{1}\right) / i_{1}\right) \cdots\left(\boldsymbol{s}\left(i_{k}\right) / i_{k}\right)}^{\boldsymbol{R}}: \boldsymbol{s} \in R^{\omega}, \boldsymbol{s} J_{*} \boldsymbol{r}\right\} \\
= & \bigwedge_{r_{1}, \ldots, r_{k} \in R} \llbracket \varphi \rrbracket_{r\left(r_{1} / i_{1}\right) \ldots\left(r_{k} / i_{k}\right)}^{\boldsymbol{R}} \\
= & \llbracket \forall v_{i_{1}} \ldots \forall v_{i_{k}} \varphi \rrbracket_{\boldsymbol{r}}^{\boldsymbol{R}} .
\end{aligned}
$$

The fact that $\forall_{J}^{\boldsymbol{F}(\boldsymbol{R})}$ and $\exists_{J}^{\boldsymbol{F}(\boldsymbol{R})}$ are defined for all $J \subseteq_{\omega} \omega$ follows at once from the assumption that $\boldsymbol{R}$ is a total $\mathcal{S}$-realization.

It follows from $(*)$ that, for any $f \in \boldsymbol{F}(\boldsymbol{R})$, if $f=\llbracket \varphi \rrbracket^{\boldsymbol{R}}$, then the set of all $i<\omega$ such that $v_{i}$ is free in $\varphi$ forms a support set of $b$. Hence $\boldsymbol{F}(\boldsymbol{R})$ is locally finite. The construction of $\boldsymbol{F}(\boldsymbol{R})$ motivates the following two definitions. 
Definition 3.3. Let $\mathcal{S}$ be a SIC over the language $\Lambda$. A polyadic algebra $\boldsymbol{A}$ is called a function-representable polyadic $\mathcal{S}$-algebra if it is isomorphic to a total functional polyadic $\left(\Lambda^{*}, I\right)$-algebra whose value algebra is of the form $\boldsymbol{V}^{*}=\left\langle\boldsymbol{V}, \forall \boldsymbol{V}^{*}, \exists \boldsymbol{V}^{*}\right\rangle$ where $\boldsymbol{V}$ is an $\mathcal{S}$-algebra and $\forall \boldsymbol{V}^{*}$ and $\exists \boldsymbol{V}^{*}$ are respectively the partial generalized meet and join operations with respect to the implication ordering of $\boldsymbol{V}$.

It is not difficult to show that every locally finite function-representable polyadic $\mathcal{S}$-algebra with coordinate set $\omega$ is of the form $\boldsymbol{F}(\boldsymbol{R})$ for some $\mathcal{S}$-realization $\boldsymbol{R}$.

In the following definition we use $x \leq y$ in axioms $\left(\mathrm{T}_{1}\right)-\left(\mathrm{T}_{7}\right)$ as an abbreviation for the equation $(x \Rightarrow y)=\top$.

Definition 3.4. Let $\mathcal{S}$ be a SIC over a language $\Lambda$ and let $I$ be a nonempty set. A polyadic $\left(\Lambda^{*}, I\right)$-algebra is called a polyadic $\mathcal{S}$-algebra if it satisfies the following identities and quasi-identities:

- Axioms $\left(\mathrm{PA}_{1}\right)-\left(\mathrm{PA}_{5}\right)$ of polyadic $\left(\Lambda^{*}, I\right)$-algebras.

- Axioms $\left(\mathrm{SA}_{1}\right)-\left(\mathrm{SA}_{4}\right)$ of $\mathcal{L}$-algebras.

- Axioms $\left(\mathrm{T}_{1}\right)-\left(\mathrm{T}_{7}\right)$ for all $\sigma \in I^{(I)}$ and $J \subseteq_{\omega} I$ :

$\left(\mathrm{T}_{1}\right) \quad x \leq y \rightarrow S_{\sigma} x \leq S_{\sigma} y$;

$\left(\mathrm{T}_{2}\right) \quad \forall \emptyset x=x$;

$\left(\mathrm{T}_{3}\right) \quad \exists_{\emptyset} x=x ;$

$\left(\mathrm{T}_{4}\right) \quad \forall_{J} x \leq x ;$

$\left(\mathrm{T}_{5}\right) \quad x \leq \exists_{J} x ;$

( $\left.\mathrm{T}_{6}\right) \quad x \leq y \rightarrow \forall_{J} x \leq \forall_{J} y$;

$\left(\mathrm{T}_{7}\right) \quad x \leq y \rightarrow \exists_{J} x \leq \exists_{J} y$.

THEOREM 3.5. Every function-representable polyadic $\mathcal{S}$-algebra is a polyadic $\mathcal{S}$-algebra.

Proof. Let $\boldsymbol{F}$ be a functional polyadic $\left(\Lambda^{*}, I\right)$-algebra with value algebra $\boldsymbol{V}^{*}=\left\langle\boldsymbol{V}, \forall \boldsymbol{V}^{*}, \exists^{*}\right\rangle$, where $\boldsymbol{V}$ is an $\mathcal{S}$-algebra. $\boldsymbol{F}$ is a subalgebra of a direct power $\left(\boldsymbol{V}^{*}\right)^{D^{I}}$ of $\boldsymbol{V}^{*}$. Thus $\boldsymbol{F}$ satisfies all the $\mathcal{S}$-algebra axioms since $\boldsymbol{V}^{*}$ does and the axioms are identities and quasi-identities. $\boldsymbol{F}$ satisfies all the polyadic algebra axioms by Thm. 1.11.

To see that $\left(\mathrm{T}_{1}\right)$ holds, let $f, g \in F$ such that $f \leq \boldsymbol{F} g$, i.e., $(f \Rightarrow \boldsymbol{F} g)=\top^{\boldsymbol{F}}$. Then $\left(S_{\sigma}^{\boldsymbol{F}} f \Rightarrow^{\boldsymbol{V}} S_{\sigma}^{\boldsymbol{F}} g\right)=S_{\sigma}^{\boldsymbol{F}}\left(f \Rightarrow^{\boldsymbol{V}} g\right)=S_{\sigma}^{\boldsymbol{F}}\left(\top^{\boldsymbol{F}}\right)=\top^{\boldsymbol{F}}$. Thus $S_{\sigma}^{\boldsymbol{F}} f \leq \boldsymbol{F} \quad S_{\sigma}^{\boldsymbol{F}} g$ and $\left(\mathrm{T}_{1}\right)$ follows.

By Def. 1.9,

$$
\left(\forall_{J}^{\boldsymbol{F}} f\right)(p)=\bigwedge\left\{f(q): q \in I^{(I)}, q J_{*} p\right\} .
$$

Hence $\left(\forall_{J}^{\boldsymbol{F}} f\right)(p) \leq \boldsymbol{V} f(p)$ for every $p \in D^{I}$, and thus $\forall_{J}^{\boldsymbol{F}} f \leq \boldsymbol{F} f$. So $\left(\mathrm{T}_{4}\right)$ holds, and by a symmetric argument so does $\left(\mathrm{T}_{5}\right)$. Similar arguments establish $\left(\mathrm{T}_{2}\right)$, $\left(\mathrm{T}_{3}\right),\left(\mathrm{T}_{6}\right)$ and $\left(\mathrm{T}_{7}\right)$. 
THEOREM 3.6. The pseudomonotonicity axiom $\left(\mathrm{PA}_{6}\right)$ holds in every locally finite polyadic $\mathcal{S}$-algebra of infinite dimension.

Proof. Let

$$
\boldsymbol{A}=\left\langle A, \Rightarrow^{\boldsymbol{A}}, \top^{\boldsymbol{A}}, O^{\boldsymbol{A}}, \forall_{J}^{\boldsymbol{A}}, \exists_{J}^{\boldsymbol{A}}, S_{\sigma}^{\boldsymbol{A}}\right\rangle_{O \in \mathcal{N}, J \subseteq_{\omega} I, \sigma \in I^{(I)}}
$$

be a polyadic $\mathcal{S}$-algebra. Its reduct $\boldsymbol{A}=\left\langle A, \Rightarrow^{\boldsymbol{A}}, \top^{\boldsymbol{A}}, O^{\boldsymbol{A}}\right\rangle_{O \in \mathcal{N}}$ is an $\mathcal{S}$-algebra, and thus the relation $\leq^{\boldsymbol{A}}$ defined by $a \leq^{\boldsymbol{A}} b$ iff $\left(a \Rightarrow^{\boldsymbol{A}} b\right)=\top^{\boldsymbol{A}}$ is the implication ordering on $\boldsymbol{A}$ and hence a partial ordering. In the remainder of the proof we omit the superscript ${ }^{\boldsymbol{A}}$.

In order to verify the pseudomonotonicity axiom for $\forall$, consider $a, b \in A$, $J \subseteq_{\omega} I$, and $\sigma, \tau \in I^{(I)}$ such that $\sigma, \tau J_{*} \iota$. Assume $\forall_{J} a=\forall_{J} S_{\sigma} b$ and $\forall_{J} b=\forall_{J} S_{\tau} a$. Let $S$ be any finite support set for $\forall_{J} S_{\sigma} b$. By Prop. 1.2 we can assume that $S \cap J=\emptyset$. Then $\tau(S)$ supports $S_{\tau} \forall_{J} S_{\sigma} b$ by Prop. 1.5. But $\tau J_{*} \iota$. So $\tau(S)=S$ and $J$ is independent of $S_{\tau} \forall_{J} S_{\sigma} b$. We also have by $\left(\mathrm{T}_{4}\right)$ that $\forall_{J} S_{\sigma} b=\forall_{J} a \leq a$. Thus

$$
\begin{aligned}
\forall_{J} S_{\sigma} b & =S_{\tau} \forall_{J} S_{\sigma} b & & \text { by }\left(\mathrm{PA}_{1}\right),\left(\mathrm{PA}_{4}\right) \text { and the fact that } \tau J_{*} \iota \\
& =\forall_{\emptyset} S_{\tau} \forall_{J} S_{\sigma} b & & \text { by }\left(\mathrm{T}_{2}\right) \\
& =\forall_{J} S_{\tau} \forall_{J} S_{\sigma} b & & \text { by Cor. } 1.3 \text { since } J \text { is independent of } S_{\tau} \forall_{J} S_{\sigma} b \\
& \leq \forall_{J} S_{\tau} a & & \text { by }\left(\mathrm{T}_{1}\right) \text { and }\left(\mathrm{T}_{6}\right) .
\end{aligned}
$$

Now applying the assumptions we have $\forall_{J} a=\forall_{J} S_{\sigma} b \leq \forall_{J} S_{\tau} a=\forall_{J} b$, and by symmetry, $\forall_{J} b \leq \forall_{J} a$.

The pseudomonotonicity of $\exists$ is verified in a similar manner.

We now give the main result of the paper.

Theorem 3.7 (Polyadic $\mathcal{S}$-Algebra Functional Representation). Every locally finite polyadic $\mathcal{S}$-algebra of infinite dimension is isomorphic to a function-representable polyadic $\mathcal{S}$-algebra.

Proof. Let

$$
\boldsymbol{A}=\left\langle A, \Rightarrow^{\boldsymbol{A}}, \top^{\boldsymbol{A}}, O^{\boldsymbol{A}}, \forall_{J}^{\boldsymbol{A}}, \exists_{J}^{\boldsymbol{A}}, S_{\sigma}^{\boldsymbol{A}}\right\rangle_{O \in \mathcal{N}, J \subseteq_{\omega} I, \sigma \in I^{(I)}}
$$

be an arbitrary locally finite polyadic $\mathcal{S}$-algebra. By Thm. 1.12, $\boldsymbol{A}$ is isomorphic to a functional polyadic $\left(\Lambda^{*}, I\right)$-algebra whose domain set is $I$, the coordinate set of $\boldsymbol{A}$, and whose value algebra is $\boldsymbol{V}^{*}=\left\langle A, \Rightarrow^{\boldsymbol{A}}, \top^{\boldsymbol{A}}, O^{\boldsymbol{A}}, \forall \boldsymbol{V}^{*}, \exists \boldsymbol{V}^{*}\right\rangle_{O \in \mathcal{N}}$, where $\forall \boldsymbol{V}^{*}, \exists^{*}: \mathrm{Sb}(A) \stackrel{\mathrm{p}}{\rightarrow} A$ are partial second-order operations whose common domain is the set of all subsets of $A$ of the form $\left\{S_{\lambda}^{\boldsymbol{A}} a: \lambda \in I^{(I)}, \lambda J_{*} \sigma\right\}$ for some $a \in A$, $J \subseteq_{\omega} I$ and $\sigma \in I^{(I)}$. Moreover, by the remarks following Thm. 1.12,

$$
\begin{aligned}
& \forall^{\boldsymbol{V}^{*}}\left\{S_{\lambda}^{\boldsymbol{A}} a: \lambda J_{*} \sigma\right\}=S_{\sigma}^{\boldsymbol{A}} \forall_{J}^{\boldsymbol{A}} a, \\
& \exists^{\boldsymbol{V}^{*}}\left\{S_{\lambda}^{\boldsymbol{A}} a: \lambda J_{*} \sigma\right\}=S_{\sigma}^{\boldsymbol{A}} \exists_{J}^{\boldsymbol{A}} a .
\end{aligned}
$$

We begin by proving that, for all $a \in A$ and $\sigma \in I^{(I)}$,

$$
S_{\sigma}^{\boldsymbol{A}_{J}}{ }_{J}^{\boldsymbol{A}} a=\bigwedge\left\{S_{\lambda}^{\boldsymbol{A}} a: \lambda J_{*} \sigma\right\},
$$




$$
S_{\sigma}^{\boldsymbol{A}} \exists_{J}^{\boldsymbol{A}} a=\bigvee\left\{S_{\lambda}^{\boldsymbol{A}} a: \lambda J_{*} \sigma\right\} .
$$

We prove only the first of these equalities; the proof of the second is completely analogous. We omit the superscript ${ }^{\boldsymbol{A}}$ to simplify notation. We observe first of all that by $\left(\mathrm{PA}_{4}\right),\left(\mathrm{T}_{1}\right)$, and $\left(\mathrm{T}_{4}\right)$, we have

$$
S_{\sigma} \forall_{J} a=S_{\lambda} \forall_{J} a \leq S_{\lambda} a \quad \text { for all } \lambda \text { such that } \lambda J_{*} \sigma .
$$

Assume now that $b \leq S_{\lambda} a$ for all $\lambda$ such that $\lambda J_{*} \sigma$. Choose $K \subseteq_{\omega} I$ such that $|K|=|J|, K \cap J=\emptyset$, and $K$ is independent of both $a$ and $b$; we also require that $\sigma K \iota$ and $\sigma^{-1}(K)=K$. Such a $K$ exists since $\boldsymbol{A}$ is locally finite and of infinite dimension by hypothesis. Consider any $\lambda$ such that $\lambda J_{*} \sigma$ and $\lambda$ maps $J$ one-to-one onto $K$. Note that $\lambda^{-1}(K)=J \cup K$ because of $\lambda J_{*} \sigma$ and the choice of $K$. Finally, let $\pi$ be any finite transformation such that $\pi K_{*} \iota$ and $\pi(K) \cap J=\pi(K) \cap K=\emptyset$. Note that $\lambda \pi(J \cup K)_{*} \sigma$. Note also that

$$
(\lambda \pi)^{-1}(K)=\pi^{-1}\left(\lambda^{-1}(K)\right)=\pi^{-1}(J \cup K)=\pi^{-1}(J) \cup \pi^{-1}(K)=J \cup \emptyset=J,
$$

and $\lambda \pi$ is one-to-one on $J$. Recall that $b \leq S_{\lambda} a$ by assumption. Thus

$$
\begin{array}{rlrl}
b & =\forall_{\emptyset} b & & \text { by }\left(\mathrm{T}_{2}\right) \\
& =\forall_{K} b & & \text { by Cor. } 1.3 \text { since } K \text { is independent of } b \\
& \leq \forall_{K} S_{\lambda} a & & \text { by }\left(\mathrm{T}_{6}\right) \\
& =\forall_{K} S_{\lambda} S_{\pi} a & & \left(S_{\pi} a=a \text { since } \pi K_{*} \iota \text { and } K \text { is independent of } a\right) \\
& =\forall_{K} S_{\lambda \pi} a & & \text { by }\left(\mathrm{PA}_{2}\right) \\
& =S_{\lambda \pi} \forall_{J} a & & \text { by }\left(\mathrm{PA}_{5}\right) \text { since }(\lambda \pi)^{-1}(K)=J \text { and } \\
& =S_{\sigma} \forall_{J} a & & \text { by is one-to-one on } J \\
& & \left(\mathrm{PA}_{4}\right) \text { since } \lambda \pi(J \cup K)_{*} \sigma .
\end{array}
$$

Thus (3) holds, and (4) is proved in a similar manner.

$\left\langle A, \Rightarrow^{\boldsymbol{A}}, \top^{\boldsymbol{A}}, O^{\boldsymbol{A}}\right\rangle_{O \in \mathcal{N}}$ is an $\mathcal{S}$-algebra because the axioms of $\mathcal{S}$-algebras are part of the axioms of polyadic $\mathcal{S}$-algebras. In view of (1)-(4), $\forall \boldsymbol{V}^{*}$ and $\exists \boldsymbol{V}^{*}$ may be taken respectively to be the generalized meet and join with respect to the implication ordering. So $\boldsymbol{A}$ is isomorphic to a function-representable polyadic $\mathcal{S}$-algebra.

Conclusion. By taking $\mathcal{S}$ to be intuitionistic logic, the many-valued logic of Łukasiewicz, modal logic (with the necessitation rule), and BCK logic, we get a functional representation theorem for the varieties of polyadic algebras over these logics. (In the case of BCK logic it is actually a quasivariety [22].) As indicated in the Introduction, these representation theorems are analogues of the functional representation theorem for locally finite Boolean polyadic algebras (of infinite degree) $[8,(10.9)]$. Some of the representation theorems found in the literature for specific logics, e.g. intuitionistic logic (Georgescu [7]) and many-valued logic 
(Schwartz [21]) correspond more closely to the stronger representation for simple, locally finite polyadic Boolean algebras [8, (17.3)]. The analogue of this stronger theorem for polyadic $\mathcal{S}$-algebras remains to be investigated. It also remains to investigate quantification over propositional logics without an underlying partial ordering, and how the theory of polyadic algebras might be extended to these logics.

\section{References}

[1] H. P. Barendregt, The Lambda Calculus. Its Syntax and Semantics, revised edition, Stud. Logic Found. Math. 103, North-Holland, Amsterdam 1985.

[2] W. J. Blok and D. Pigozzi, Algebraizable logics, Mem. Amer. Math. Soc. 396 (1989).

[3] Z. B. Diskin, Polyadic algebras for non-classical logics, I,II,III,IV, Latv. Mat. Ezhegodnik (in Russian), to appear.

[4] J. Freedman, Algebraic semantics for modal predicate logic, Z. Math. Logik Grundlag. Math. 22 (1976), 523-552.

[5] G. Georgescu, A representation theorem for tense polyadic algebras, Mathematica (Cluj), 21 (1979), 131-138.

[6] —, Modal polyadic algebras, Bull. Math. Soc. Sci. Math. R. S. Roumanie (N.S.) 23 (1979), 49-64.

[7] —, A representation theorem for polyadic Heyting algebras, Algebra Universalis 14 (1982), 197-209.

[8] P. Halmos, Homogeneous locally finite polyadic Boolean algebras of infinite degree, Fund. Math. 43 (1956), 255-325; see also [9], pp. 97-166.

[9] — Algebraic Logic, Chelsea, New York 1962.

[10] L. Henkin, An algebraic characterization of quantifiers, Fund. Math. 37 (1950), 63-74.

[11] L. Henkin, J. D. Monk and A. Tarski, Cylindric Algebras, Parts I and II, NorthHolland, Amsterdam 1971 and 1985.

[12] J. Kotas and A. Pieczkowski, On a generalized cylindrical algebra and intuitionistic logic, Studia Logica 17 (1966), 73-80.

[13] A. R. Meyer, What is a model of the lambda calculus?, Inform. Control 52 (1982), 87-122.

[14] D. Monk, Polyadic Heyting algebras, Notices Amer. Math. Soc. 7 (1960), 735.

[15] A. Mostowski, Proofs of non-deducibility in intuitionistic functional calculus, J. Symbolic Logic 13 (1948), 204-207.

[16] I. Németi, Algebraizations of quantifier logics. An introductory overview, Studia Logica 50 (1991), 485-569.

[17] D. Pigozzi and A. Salibra, The abstract variable-binding calculus, manuscript.

[18] —, - - An introduction to lambda abstraction algebras, manuscript.

[19] H. Rasiowa, An Algebraic Approach to Non-Classical Logics, North-Holland, Amsterdam 1974 .

[20] H. Rasiowa and R. Sikorski, The Mathematics of Metamathematics, PWN, Warszawa 1963.

[21] D. Schwartz, Polyadic MV-algebras, Z. Math. Logik Grundlag. Math. 26 (1980), 561564.

[22] A. Wroński, BCK-algebras do not form a variety, Math. Japon. 28 (1983), 211-213. 